\section{Sleep and Breathing}

\section{Helga Peter}

Marburg, Deutschland

\section{Definition}

Zeitschrift.

Siehe $\triangleright$ „Schlafmedizinische Fachgesellschaften, Fachzeitschriften und Publikationsforen“". 